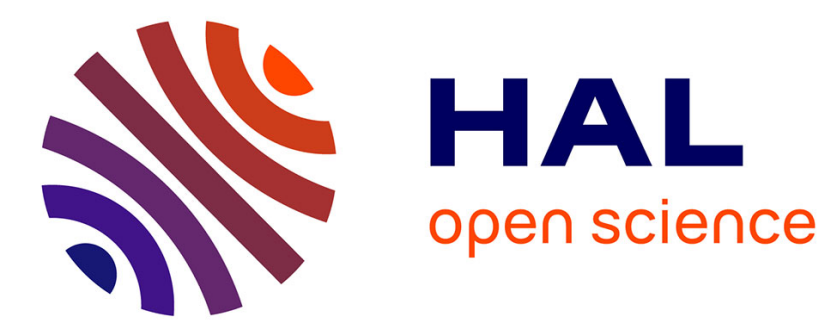

\title{
Le financement des services essentiels dans les villes pauvres
}

Pierre-Noël Giraud, Benoît Lefèvre, Augustin Maria, Joël Ruet

\section{To cite this version:}

Pierre-Noël Giraud, Benoît Lefèvre, Augustin Maria, Joël Ruet. Le financement des services essentiels dans les villes pauvres. Revue d'économie financière, 2006, 86 (5), pp.285-308. hal-00871468

\section{HAL Id: hal-00871468 https://hal.science/hal-00871468}

Submitted on 9 Mar 2015

HAL is a multi-disciplinary open access archive for the deposit and dissemination of scientific research documents, whether they are published or not. The documents may come from teaching and research institutions in France or abroad, or from public or private research centers.
L'archive ouverte pluridisciplinaire HAL, est destinée au dépôt et à la diffusion de documents scientifiques de niveau recherche, publiés ou non, émanant des établissements d'enseignement et de recherche français ou étrangers, des laboratoires publics ou privés. 


\section{$\begin{array}{lllll}\text { C } & \mathbf{E} & \mathbf{R} & \mathbf{N} & \mathbf{A}\end{array}$}

Cerna, Centre d'économie industrielle Ecole Nationale Supérieure des Mines de Paris / ParisTech

60 , boulevard Saint Michel 75272 Paris Cedex 06 - France Tél. : 33 (1) 40519091 - Fax : 33 (1) 44071046 pierre-noel.giraud@ensmp.fr - http://www.cerna.ensmp.fr

\section{Le financement des services essentiels dans les villes pauvres}

Pierre-Noël Giraud, Benoit Lefevre, Augustin Maria (Cerna), Joël Ruet (London School of Economics)

Article publié dans :

Revue d'Economie Financière

Automne 2006, Paris 
1) Delhi : un état des lieux emblématique _ 5

Eau et assainissement _6

Electricité —7

Transport 8

Conclusion _9

2) Qui a droit aux services essentiels publics ? 99

Propriété foncière et accès aux services à Delhi__ 9

Le grand saut de la politique urbaine au Maroc _ 11

3) Les Partenariats Publics Privés (PPP) 12

Des espoirs mal placés _ 12

Saisir le bon maillon pour briser le cercle vicieux de la dégradation des services _ 13

4) Que faire dans les quartiers pauvres?

\begin{tabular}{|l|l|}
\hline Les réseaux condominiaux. 15 \\
\hline
\end{tabular}

\begin{tabular}{ll}
\hline Le programme INDH à Casablanca __ & 17 \\
\hline
\end{tabular}

5) Les riches font sécession : est ce si grave ?

\begin{tabular}{|ll|}
\hline Lutter contre l'automobile en ville__ 19 \\
\hline
\end{tabular}

Encadrer les innovations dans les systèmes décentralisés __ 20

6) Fiscalité locale et financement des infrastructures de services

\begin{tabular}{ll}
\hline essentiels & 20 \\
\hline
\end{tabular}

\begin{tabular}{|ll}
\hline Conclusions 23 \\
\hline
\end{tabular}

\begin{tabular}{|ll|}
\hline Références 25 & 25 \\
\hline
\end{tabular} 


\section{Introduction}

Les grandes villes des pays pauvres et émergents présentent les caractéristiques suivantes.

1) Elles sont en croissance rapide. L'ONU estime que la population urbaine de ces pays augmentera de deux milliards en 30 ans. Cette explosion urbaine exigera d'importants investissements, appelés par Michel Arnaud et Jean-Marie Cour des «investissements de peuplement » (Cour, 2005). Ils concernent essentiellement : les bâtiments, privés et publics, et les infrastructures des réseaux qui fournissent les services urbains essentiels: eau, assainissement liquide et solide, électricité, voirie et transports publics, télécommunications.

2) À cet horizon, 30ans, il est exclu, quoi qu'en disent de nombreuses déclarations gouvernementales, de pouvoir «éradiquer» les bidonvilles. Une large part de la population restera très pauvre et devra se contenter d'habiter dans le genre de quartiers que l'on qualifie aujourd'hui «d'informels » ou «d'illégaux ». C'est une qualification juridique. Nous verrons que la « légalisation» de ces quartiers est un enjeu décisif de la fourniture des services essentiels à ceux qui y vivent. Cependant, même légalisés, ils resteront pauvres, avec une majorité de leur population faisant partie du secteur également dit « informel» et qualifié plus justement par d'autres « d'économie populaire».

3) À l'autre bout du spectre social urbain, des classes moyennes et riches, directement ou indirectement connectées à l'économie globale, se développent rapidement, surtout dans les pays émergents en croissance rapide, moins vite dans les pays les plus pauvres. Elles ont déjà et auront de plus en plus tendance à vouloir s'autonomiser du reste de la ville, en particulier de ses parties pauvres, par exemple en revendiquant pour elles seules, quitte à les financer elles mêmes, des services urbains efficaces, garant de leur compétitivité et d'un mode de vie conforme à leurs revenus croissants.

Lieu d'un inégalité sociale et spatiale croissante, duales, fragmentées, telles sont et seront, si de vigoureuses politiques publiques ne viennent pas s'y opposer, les grandes villes des pays pauvres et émergents.

Dans ces villes, la fourniture des services urbains essentiels est généralement, dans le meilleur des cas, à peine satisfaisante pour les classes moyennes et les riches et de très mauvaise qualité, 
voire inexistante, pour les plus pauvres. Un cas moins favorable, hélas très répandu dans nombre de villes émergentes, est que les organismes prestataires des services essentiels, parce qu'ils sont très mal incités et gérés, ne parviennent même pas à satisfaire la demande solvable qui s'adresse à eux. Il en est, par exemple, souvent ainsi dans des lotissements pour classes moyennes construits en grande banlieue.

Une question préalable est bien sûr: «faut il que la puissance publique et en particulier les municipalités se soucient de ce que les pauvres n'ont pas un accès suffisant aux services essentiels»? «Oui », répondra-t-on, si la non consommation de services essentiels par les pauvres engendre des externalités négatives (une mauvaise eau plus des déchets non collectés engendrent des épidémies, des déchets non collectés et l'absence d'assainissement entraînent une pollution de la nappe, par exemple). «Oui» encore, si la municipalité juge nécessaire une politique de réduction de la pauvreté et identifie la fourniture de services essentiels comme un moyen efficace de cette politique. On peut en effet soutenir que l'absence ou la mauvaise qualité des services essentiels annule en réalité pour les plus pauvres tout l'intérêt économique de la ville, qui, pour eux comme pour tous, réside dans la densité des interactions économiques et sociales permises par la ville. Autrement dit, la fourniture des services essentiels serait alors l'un des principaux moyens de lutter contre les « trappes à pauvreté » urbaines.

Mais on répondra «non» dans les cas contraires. On ferra alors remarquer que les plus pauvres se débrouillent toujours en ville pour trouver des solutions à la mesure de leurs faibles revenus. C'est l'enrichissement de la ville et lui seul qui peut les tirer de leur pauvreté et leur donner accès à des services de meilleure qualité. Tous les moyens de la puissance publique devraient donc être utilisés à promouvoir la croissance économique maximum de la ville, sans se soucier de politiques spécifiques à l'égard des plus pauvres.

Comme ce débat, dont le contenu économique est réel mais complexe, ne peut ultimement être tranché qu'au plan politique, car il s'agit en fait de choisir le niveau d'inégalité politiquement tolérable dans une ville, nous n'y entrerons pas ici. Nous prendrons comme hypothèse que la municipalité et le gouvernement, quelle qu'en soient les raisons, se sont fixé des objectifs d'extension d'une offre de services essentiels économiquement abordables par les plus pauvres. Comment financer des services urbains qui soient accessibles aux plus pauvres, quand les riches qui peuvent payer l'extension des réseaux sont tentés de faire sécession? Telles sont les données du problème.

Sa solution exige des innovations, car le modèle qui a conduit en un siècle à l'équipement complet des villes européennes et américaines ne peut à l'évidence être reproduit à l'identique dans les villes du sud aujourd'hui. En effet, dans les pays aujourd'hui riches, l'urbanisation fût 
en général moins rapide. Ensuite, une solidarité objective entre habitants de la ville (à la fin du $19^{\text {éme }}$ et au début du $20^{\text {ème }}$ siécle, les riches urbains pouvaient plus difficilement «s'isoler» physiquement des plus pauvres) a permis d'imposer des subventions croisées, entre riches et pauvres et entre services. Enfin l'inflation, le plus extraordinaire instrument de transfert de revenus jamais inventé, s'est avérée une forme très commode de subvention générale de l'ensemble de ces secteurs par les rentiers. Ces conditions ne sont plus réunies dans les actuels pays pauvres et émergents. En revanche, l'évolution technique permet d'envisager aujourd'hui, pour desservir les quartiers les plus pauvres, des systèmes moins coûteux et cependant aussi, voir plus efficaces que les systèmes copiés des villes riches.

En section 1 nous brossons à grands traits un tableau de la situation des services essentiels à Delhi en Inde, une situation emblématique des pays émergents qui permet d'illustrer les problèmes posés. En section 2, à partir des exemples de l'Inde et du Maroc, nous soulignons l'importance et le caractère préalable des questions juridiques et politiques : il est d'abord nécessaire de reconnaître pleinement l'existence des quartiers et de l'habitat illégaux, de « régulariser l'illégalité », ou pour faire une analogie avec la situation des « sans papiers » dans les villes riches, de «régulariser les sans citoyenneté urbaine». Puis, nous abordons, à partir d'études de cas variées, les solutions qui nous paraissent les plus prometteuses et qui passent toutes, c'est la thèse que nous défendons, par des innovations non seulement organisationnelles mais aussi techniques. En section 3, nous faisons un rapide bilan des partenariats publics privés (PPP), aujourd'hui moins en vogue, mais qui gardent un potentiel certain d'amélioration de l'efficacité de la fourniture des services, si l'on sait les adapter. En section 4, nous donnons des exemples de politiques spécifiques concernant les quartiers pauvres, en particulier celles qui recherchent un abaissement des coûts par des techniques innovantes. En section 5, nous abordons les stratégies d'autonomisation des riches en nous demandant si le mouvement ne peut être intelligemment accompagné. La section 6 aborde brièvement la fiscalité locale.

\section{1) Delhi : un état des lieux emblématique}

La capitale fédérale de l'Inde rassemble 15,4 millions d'habitant, sur une surface de $1483 \mathrm{~km}^{2}$. Le taux de croissance de la population se situe autour de $4 \%$ par an dont à peine un tiers provient de l'exode rural. Le revenu annuel moyen est d'environ 50000 Rs (1000 €) par habitant. En 2000, le gouvernement de l'état de Delhi annonçait un taux de pauvreté légèrement inférieur à 9\% (le seuil officiel de pauvreté est fixé à Delhi à 454 Rs par personne et par mois, soit 0,3 US $\$$ par jour et par personne au taux de change courant). Les densités sont très 
variables selon les quartiers, allant de densités très élevées à l'intérieur des remparts de Old Delhi (plus de 1000 personnes par hectare) à des densités plus faibles dans l'ancienne Delhi coloniale. On assiste à un rapide développement de villes périphériques dont le raccordement aux réseaux centraux pose problème.

\section{Eau et assainissement}

Une régie municipale, le Delhi Jal Board (DJB) est responsable des services d'eau et d'assainissement liquide, sauf évidemment, comme on le verra ci-dessous, dans les quartiers sans existence juridique. La quasi-totalité des résidents de logements appartenant à la partie formelle du parc sont connectés par branchements individuels au réseau de distribution d'eau, ce qui représente 1.33 millions de branchements. Cependant, le service est très dégradé en raison de l'incapacité de la régie à assurer une distribution permanente : horaires de distributions souvent peu commodes (une heure tôt le matin et une heure le soir), et problèmes de qualité inhérents à la distribution par intermittence, qui permet l'infiltration d'eaux polluées dans le réseau de distribution lorsqu'il n'est pas sous pression.

Face à cette situation, les ménages mettent en œuvre des stratégies de réponse. Zerah (Llorente M. et Zérah M.-H., 2003) a réalisé une enquête en 1997 auprès de ménages connectés au réseau d'approvisionnement public. Les résultats de son enquête montrent que, pour un montant annuel moyen de la facture de 336 Rs $(6,7 €)$, le coût annuel moyen de l'ensemble des mesures prises (désinfectants, filtres, réservoirs, etc....) à titre privé pour faire face à l'intermittence de l'approvisionnement et aux problèmes de qualité s'élève à 2087 Rs (42 €). La contribution directe des ménages connectés au financement du service public est donc plus de cinq fois inférieure aux dépenses privées destinées à compenser les insuffisances du service public. Dutta et. al. (2005), sur la base d'une enquête réalisée en 2002 sur un échantillon incluant également les ménages non connectés au réseau (environ un quart du total), arrive à un coût annuel moyen de 4533 Rs $(90 €)$. L'enquête montre également que les dépenses de compensations augmentent avec le standing du quartier. Ainsi, les dépenses annuelles moyennes dans les quartiers les plus modestes sont de 2525 Rs, alors qu'elles sont de 6000 Rs dans les quartiers les plus aisés. Ce résultat pousse à dépasser le constat habituel qui consiste à dire que "les pauvres payent plus car ils ne sont pas connectés au réseau". En réalité, les plus riches payent également des sommes conséquentes pour améliorer le service qu'ils reçoivent de la régie publique.

L'adaptation des usagers à la mauvaise situation de l'approvisionnement par le réseau public pousse également au développement de filières d'approvisionnement privées. Ces filières peuvent prendre la forme d'initiatives individuelles comme l'investissement dans un forage indépendant. Elles peuvent également être organisées par des investisseurs privés sous la forme 
d'entreprises d'approvisionnement par camion citerne, ou de mini réseaux desservant des quartiers informels non connectés au réseau mais abritant des populations solvables qui souhaitent avoir accès à un approvisionnement individuel à domicile. La multiplication de ces filières parallèles, souvent basées sur l'exploitation de la ressource souterraine locale, engendre une dégradation rapide de cette ressource alors même que son rôle dans l'approvisionnement de la ville prend une importance tous les jours croissante, jusqu'à atteindre, en terme de volume, la contribution de l'approvisionnement par le réseau. (Maria, 2004)

\section{Electricité}

A Delhi, le taux de connexion des ménages au réseau électrique est proche de $100 \%$. Mais, comme dans le cas de l'eau, les problèmes viennent de la qualité de fourniture. Les coupures sont très fréquentes et contraignent les utilisateurs qui ne peuvent les supporter à s'équiper en moyens d'autoproduction.

Ville relativement riche, Delhi aurait les moyens de payer son électricité à son coût complet de developpement. Mais le problème principal est de gestion : les pertes énergétiques totales de la seule distribution sont de l'ordre de 54\%, avec une variation de 40 à $60 \%$ environ selon les trois zones de distribution, et des pointes locales à $90 \%$ dans les zones pauvres (pour un montant en valeur, il est vrai, très faible dans ces zones). Ces pertes comprennent des pertes techniques de l'ordre de 17 à $18 \%$ selon les zones, le solde constituant un important réservoir d'amélioration de «pertes non techniques» (vols, énergie non comptée). Avant la privatisation de la distribution, cette mauvaise gestion engendrait pour le «Delhi Electricity Board» (DEB), organisme public et verticalement intégré de production-transport-distribution d'électricité, des pertes financières annuelles de $72 \%$ de la valeur de ses actifs.

En 2002, le DEB a en effet subi une désintégration verticale entre trois types d'entités différentes. La production est restée publique, mais le recours à des producteurs privés par les opérateurs de distribution est autorisé. Le transport est resté public et la distribution a été séparée en trois zones et privatisée. Deux des trois zones ont été reprises par une filiale du groupe Reliance, l'autre étant reprise par une filiale du groupe Tata, les deux plus grands groupes industriels privés indiens. Devant l'incertitude sur l'état réel du réseau et donc sur les coûts d'une amélioration de la distribution, les repreneurs privés ne se sont engagés que sur des réduction des pertes (c'était un des critères de l'appel d'offre) assez faibles: de 0.5 à $3 \%$ d'amélioration annuelle, quant le réglementeur en attendait de 1.5 à $6 \%$, et ils ont proposé une valeur d'achat des actifs de moins du quart de la valorisation par les experts. Enfin l'Etat de Delhi a du s'engager à financer lui-même l'essentiel des investissements dans le réseau, du moins dans une première période. En 2006, le bilan est contrasté : la privatisation a très 
lentement mais certainement commencé d'améliorer l'efficacité de la distribution. Mais, les investissements ayant été financés par l'Etat, le rôle du secteur privé s'est borné à améliorer la gestion, ce qui, il est vrai, était bien le problème essentiel.

\section{Transport}

Le nombre de véhicules à Delhi est passé de 2,24 millions en 1994 à 4,18 millions en 2004, soit un taux de croissance annuel de 6,5\%. Cette explosion est principalement engendrée par la motorisation croissante des riches: le nombre de voitures et de deux-roues individuelles a augmenté respectivement de $150 \%$ et $78 \%$ en dix ans. Représentant $1 \%$ du parc total de véhicule, les bus supportent $60 \%$ des déplacements. Les véhicules particuliers (64\% du parc) satisfont $30 \%$ de la demande de transport. Malgré un réseau routier particulièrement développé (1749 Km de route pour $100 \mathrm{Km} 2$, soit plus de 20 fois la moyenne nationale), l'offre d'infrastructure routière ne parvient pas à suivre la demande. Il en résulte une forte congestion, une augmentation des coûts et temps de transport et un niveau de pollution local et global dramatique.

La compagnie de transport publique, le Delhi Transport Corporation (DTC), perd chaque mois 5 millions $€$. Le ratio employés / bus est de 12 au lieu de 6, norme internationale. Dans ces conditions, la DTC ne peut améliorer, et encore moins étendre son service. En 2003 une étude d'un consultant indien a cependant permis de corriger certains disfonctionnements dans la gestion des opérations : le taux d'utilisation de la flotte de bus est ainsi passé de $61 \%$ à $86 \%$.

Jusqu'à récemment essentiellement limitée à l'augmentation de la capacité du réseau routier par la construction de «ring roads", «flyovers» et autoroutes urbaines, la stratégie de la municipalité était une pure fuite en avant, d'autant plus dangereuse qu'elle engendrait une structure urbaine étalée, de plus en plus dépendante de la voiture. Les scénarios «au fil de l'eau » prévoient une augmentation de 0,8 à 1,2 du nombre de déplacement par personne et par jour d'ici 2021, et une croissance de 50\% des longueurs moyennes de trajet (voiture comme bus). En réponse le nombre de véhicules passerait à 8 millions.

Pour lutter contre cette tendance, la ville s'est lancée dans un ambitieux projet de métro dont le coût final de la première phase - plus de 2 milliards d' $€$ - est plus de deux fois supérieur au coût initialement prévu. Inaugurées en septembre 2005, les trois premières lignes sont aujourd'hui relativement sous utilisées. L'absence d'infrastructure servant l'inter modalité (park\&ride, station de taxi et rickshaw, intégration avec le réseau de bus) et d'un réseau performant d'alimentation par bus expliquerait cet échec temporaire. Malgré d'intenses discussions, un projet sérieux de « Bus Rapid Transit » (BRT) peine à émerger. 


\section{Conclusion}

Le constat est accablant : les services municipaux fonctionnent de moins en moins bien. Ils ne sont même pas capables de satisfaire la demande solvable des classes moyennes, a fortiori les plus pauvres. La ville s'étale est dépend de manière croissante de l'automobile. Tous les habitants, les riches comme les pauvres, sont contraints d'adopter diverses stratégies d'adaptation ayant recours au secteur privé, qui sont en général plus coûteuses que ce que pourrait, en théorie, offrir un service public efficace.

En première analyse, le problème n'est donc pas principalement financier. En effet, les habitants payant déjà plus cher que le tarif actuel des services publics de mauvaise qualité, il existe un consentement à payer pour l'amélioration de ces services. Mais le tarif ne couvre en général même pas les coûts d'opération et de maintenance du service public, et donc ce service continue de se dégrader.

On voit bien que la ville et ses habitants sont ainsi pris dans un cercle vicieux. Il s'agit avant tout de comprendre quel est le bon maillon à attaquer pour le briser. Avant d'y venir, il nous faut au préalable souligner que, pour offrir des services à tous il faut aussi et même d'abord traiter une question juridique et politique. Tous dans la ville sont en effet loin d'être juridiquement égaux devant le service public...

\section{2) Qui a droit aux services essentiels publics ?}

\section{Propriété foncière et accès aux services à Delhi}

En nous limitant ici, à titre d'exemple, aux services de l'eau et d'assainissement, voici comment se repartit la population des 14 millions d'habitants de Delhi quant à l'accès à ces services :

Répartition de la population par forme d'habitat et accès aux services de l'eau à Delhi.

\begin{tabular}{llllll}
\hline Type d'établissements & $\begin{array}{l}\text { Population** } \\
\text { (millions) }\end{array}$ & $\begin{array}{l}\text { Population } \\
(\% \text { du total) }\end{array}$ & $\begin{array}{l}\text { Situation } \\
\text { foncière }\end{array}$ & Pauvreté** & Accès aux services \\
\hline «JJ clusters » (squatters) & 2.07 & 14.84 & Illégale & Forte & Aucun droit
\end{tabular}


Colonies de repeuplement (où sont relogés les expulsés de 1.78

l'illégal)

Colonies non autorisées et non régularisées

Colonies non autorisées mais régularisées

Slums (très fortes densités, 2.66

essentiellement : « Old Delhi)

Villages ruraux

Villages urbains

0.89

Colonies planifiées (par la DDA) 3.31

TOTAL population, millions

13.96
Légale

Forte

19.08

5.3

6.36

23.69

100
Droit de principe souvent non respecté

(retard dans la mise en œuvre)

$\begin{array}{lll}\text { Semi } & - & \\ \text { légale } & & \text { Variable Aucun droit }\end{array}$

Droit à l'accès soumis au paiement d'un forfait de développement

Restreint par des obstacles techniques

N'est pas sous la responsabilité du DJB

Bonne situation

Bonne situation

Source: Augustin Maria, d'après :

* Delhi Economic Survey 2003-2004, Planning Department, Government of National Capital Territory Delhi.

** Jain AK, (1990) Making of a Metropolis: Planning and Growth of Delhi, New Delhi:

\section{National Book Organisation}

On voit dans ce tableau que plus de $25 \%$ de la population n'a simplement aucun droit aux services de l'eau. Officiellement, ces gens n'ont aucune existence aux yeux du DJB qui gère l'eau dans la ville. Le nombre de ceux qui ont légalement droit aux services de l'eau, mais qui en pratique sont peu ou pas desservis, représente un autre quart de la population. Le problème foncier est donc fondamental pour l'accès aux services essentiels, on le sait parfaitement depuis longtemps. Sans droits fonciers, les habitants sont volontairement ignorés des services publics, car les desservir et leur donner des factures reviendrait à régulariser de fait leur situation foncière. Or l'administration ne veut pas le faire, car elle poursuit officiellement la chimère qu'elle va réussir à maîtriser la croissance urbaine et à « éradiquer » l'informel.

Les droits des résidents informels ne sont cependant jamais totalement absents, ils font l'objet d'une ambiguïté qui reflète la tension fondamentale entre le développement planifié de la ville et 
son développement spontané. Ainsi, leur situation administrative est celle d'étrangers à la ville, ils ne sont pas considérés comme des résidents à part entière, cependant en Inde, ils ont le droit de vote, ainsi qu'une certaine reconnaissance de la durée d'occupation par le biais de cartes de rationnement. Si la carte de rationnement («Ration Card») est le document qui permet aux résidents de ces quartiers illégaux d'avoir droit à une parcelle à la périphérie de la ville en cas d'éviction forcée, c'est leur carte d'électeur qui entre en jeu dans leur stratégie d'accès à l'eau. Et c'est ce qui permet de comprendre leur rationalité: n'étant pas «régularisés », ils refusent de contribuer financièrement à un service qu'ils savent pouvoir obtenir malgré tout, sous forme minimale (des bornes fontaines par exemple), par le biais des fonds de développement mis à la disposition de leurs élus, à des fins électorales, par la municipalité ou le gouvernement de l'Etat.

\section{Le grand saut de la politique urbaine au Maroc}

En avril 2005 le roi du Maroc, tirant les leçons de l'échec de 30 ans de politique de « résorption de l'habitat insalubre », et sans doute inquiet de la progression des partis islamistes parmi les pauvres, a lancé « l'Initiative Nationale de Développement Humain » (INDH). Dans le cadre de cette initiative, il est demandé aux régies municipales ainsi qu'aux deux entreprises mixtes, filiales des deux grands groupes français de l'eau, qui gèrent les services de l'eau, de l'assainissement et de l'électricité dans les principales villes du Maroc (Casablanca, Rabat Salé, Tétouan, Tanger), de présenter des plans pour connecter d'ici 2015 à l'eau, l'électricité et l'assainissement tous les ménages, sans exception et quel que soit leur statut foncier.

Le tabou de la régularisation massive de l'informel a donc été levé. Cette expérience mérite d'être suivie avec intérêt. En effet, en reconnaissant officiellement que le seul avenir des bidonvilles n'est pas d'être ignorés avant d'être détruits, mais qu'ils peuvent aussi faire l'objet d'opérations de restructuration et d'équipement en gardant sur place l'essentiel de leur population, l'initiative exige ainsi que soient simultanément accélérés : 1) le passage d'un droit du sol, essentiellement encore traditionnel, à un droit moderne assis sur l'établissement d'un cadastre, 2) la définition de nouvelles normes réalistes d'urbanisme et d'habitat applicables aux quartiers pauvres à restructurer, pour qu'ils deviennent des sortes de «médinas» contemporaines à forte densité. A Casablanca par exemple, 150000 ménages sont concernés, soit un million d'habitants.

En conclusion, un préalable au développement de programmes d'accès de tous à des services urbains de qualité acceptable est que tous les habitants soient administrativement et politiquement considérés comme faisant partie de la ville, ce qui n'est en général jamais le cas. En la matière, énormément de progrès reste à faire, de la part des gouvernements centraux et municipaux, pour traiter cette question avec pragmatisme et sortir des illusions qui prétendent 
qu'on pourra éradiquer les bidonvilles en deux ou trois décennies. Les bidonvilles et autres quartiers pauvres illégaux font partie pour longtemps des villes émergentes. Le premier pas consiste à le reconnaître. Reste à savoir comment financer leur équipement en infrastructures de services essentiels.

\section{3) Les Partenariats Publics Privés (PPP)}

\section{Des espoirs mal placés}

On a mis, il y a une dizaine d'années, de grands espoirs dans les partenariats publics privés (PPP) pour financer l'extension à tous des services essentiels. Or il est clair que, sur le plan théorique, la nature publique ou privée de l'opérateur des services ne change en rien la question du financement. Le financement est une question d'une part de tarifs et de subventions (croisées ou pas), et d'autre part d'accès des entités qui développent les réseaux à des ressources financières longues et si possible bon marché (AFD, 2005).

De ce strict point de vue, il n'y avait a priori rien à attendre de l'intervention des firmes internationales de l'eau, de l'électricité, des transports et des télécommunications dans les pays pauvres et émergents. Il leur faut emprunter en monnaie locale si elles veulent financer des développements de réseaux sans prendre de risques de change difficiles à couvrir (ce qu'elles n'ont pas toujours fait, avec les désastreuses conséquences que l'on sait, en particulier en Amérique latine). Or elles n'ont sur ce point aucun avantage décisif par rapport à des firmes privées locales et à ou des institutions financières spécialisées dans le financement des municipalités.

Si l'on pensait que les PPP internationaux pouvaient être utiles au financement de l'extension des réseaux et des services, c'est aussi parce qu'on les voyait comme $\boldsymbol{l} \boldsymbol{e}$ moyen d'améliorer la gestion des services, et de dégager ainsi des marges suffisantes pour en financer en partie l'extension. Face aux difficultés à réformer les lourdes bureaucraties qu'étaient devenues les services municipaux, le recours au secteur privé est apparu, en particulier à la Banque Mondiale, comme seul capable de briser les cercles vicieux décrits dans le cas de Delhi et d'y substituer le cercle vertueux suivant. Améliorer la gestion du service public par des investissements minima de maintenance et surtout une profonde réforme des modes de gestion, de manière à obtenir des économies rapides par réduction des pertes techniques et non techniques. Ruet (Ruet, 2006 b) montre ainsi par exemple qu'à Delhi, même à tarifs constants, la rationalisation de la 
maintenance et de l'utilisation des centrales conduit à un taux de rentabilité interne de $116 \%$ et la diminution des pertes techniques à un taux de $27 \%$. Réinvestir aussitôt ces économies dans l'amélioration de la qualité du service a ceux qui sont déjà connectés, rendant ainsi inutiles leurs coûteuses stratégies de compensation et donc acceptable par eux une augmentation des tarifs. Cette augmentation permet à son tour permet de financer des investissements plus importants pour une nouvelle amélioration de la qualité de service et peut être de financer le développement des réseaux pour qu'ils atteignent les plus pauvres, etc. Ce dernier point reste cependant problématique et nous y reviendrons.

Force est de constater que le caractère étranger et privé des grandes firmes de l'eau, de l'électricité et même des transports (bien que dans ce domaine, comme dans les télécommunications, la présence du privé est plus facilement tolérée) ne leur a pas facilité la tâche dans de nombreux pays. Alors que la seule question sérieuse est celle des meilleurs moyens de fournir les services essentiels au plus grand nombre au plus bas coût possible (en incluant les coûts environnementaux et sociaux), un curieux débat s'est déployé avec les «alter mondialistes » sur la question de savoir si l'accès à l'eau et à l'électricité était un «droit de l'homme » ou un « service marchand», alors qu'il est évidemment les deux. Les partisans de la thèse des droits font à juste titre valoir que les firmes privées ne sont pas conçues pour satisfaire des droits de l'homme, mais plutôt pour faire du profit en vendant des services. Ils se sont donc opposés fermement aux PPP, en particulier avec les «multinationales ». La bonne question est cependant simplement de savoir si, et dans quelles conditions, une firme privée peut être plus efficace, dont abaisser le coût social total du service, qu'une institution publique.

\section{Saisir le bon maillon pour briser le cercle vicieux de la dégradation des services}

Mais la véritable raison des échecs constatés (en dehors de la non couverture des risques de change) tient sans doute à une mauvaise compréhension du bon maillon à saisir pour enclencher le cercle vertueux de la réforme du prestataire de service que nous avons décrit ci dessus. Il faut en effet d'abord améliorer le service et ensuite, une fois cette amélioration reconnue par les usagers, augmenter les tarifs, et non augmenter d'abord les tarifs pour financer l'amélioration du service. Car il est un fait incontestable dont il faut toujours partir : en général les gens, mêmes les plus pauvres, payent pour des services de mauvaise qualité. Et ils les paient cher, en argent, avec leur temps, en termes de perte de santé. Ils sont toujours prêts à payer le même prix pour un service « public » meilleur, et ils sont tout simplement enthousiastes à l'idée de payer moins cher un meilleur service. Tous les exemples le prouvent. Les entreprises qui l'ont compris et pratiqué ont en général réussi à surmonter les obstacles politiques et idéologiques à l'intervention du secteur privé, surtout étranger, dans la fourniture de services à juste titre 
considérés comme essentiels.

Il s'agit donc d'une question d'ordonnancement des réformes. La "privatisation » brutale d'entités publiques très mal gérées n'est jamais la solution. Ce n'est qu'un moyen pour les autorités publiques de se débarrasser d'un problème qu'elles ne savent pas résoudre. L'échec est certain. Dans ces conditions, une phase d'amélioration de la gestion, sous forme de contrat de services par exemple, ou l'entité privée ne se paye que sur les améliorations de recouvrement des factures, la réduction des pertes, etc., est indispensable pour enclencher le cercle vertueux. Ruet (2006) a proposé de combiner cette prestation de service de gestion avec une option (au sens financier d'une option d'achat) sur la phase suivante, la délégation de service public avec prise en charge de l'exécution de travaux plus lourds de rénovation et d'extension. Ainsi, selon Ruet, la bureaucratie inefficace qu'est devenue le service public doit d'abord être « entreprisée », tout en restant publique, avant d'envisager éventuellement une privatisation. «Entrepriser» signifie doter le service public de systèmes d'information et de dispositifs de gestion internes qui soient ceux des «entreprises publiques» efficaces, et non ceux d'administrations fonctionnant dans une tout autre logique.

Une fois les services publics ainsi réformés, on s'aperçoit d'ailleurs que le spectre des articulations possibles et efficaces entre public, privé international, privé national, petit privé local et organisations de la société civile, est beaucoup plus large qu'on ne l'imagine généralement. Mais la réforme du service public est un préalable à l'exploitation du potentiel de ces partenariats (Ruet, Saravanan, Zerah, 2002).

En matière de PPP, les exemples de réussite sont plus nombreux dans le secteur des transports urbains et des télécommunications, car l'amélioration du service y est en général plus facile à démontrer rapidement. C'est le cas, par exemple, du désormais emblématique «Bus Rapid Transit» Transmilenio à Bogota. Mais, malgré de nombreux retraits, il en existe aussi dans l'eau et l'électricité. Ainsi au Maroc et dans certains pays d'Afrique de l'ouest. De manière générale, on découvre aussi de manière croissante, au delà des multinationales, le rôle que peut jouer le secteur privé local, y compris le secteur « informel ».

\section{4) Que faire dans les quartiers pauvres ?}

On l'a dit, d'abord reconnaître leur existence, c'est le premier tabou à briser. Mais il en est un autre, celui de «l'égalité technologique ». Pour beaucoup, et en particulier pour les ingénieurs à la tête des services publics et pour les hommes politiques, user de technologies différentes dans 
les quartiers riches et pauvres est une hérésie. Selon eux, tout le monde a droit à la même chose et il est inadmissible de dégrader les normes de sécurité et de qualité de service dans les quartiers pauvres. Cela part certainement d'un bon sentiment: "refuser la société à deux vitesses ». En pratique, combiné avec le premier tabou, la «régularisation de l'illégal », cela conduit à condamner les pauvres à acheter, par exemple, une eau non potable à des entrepreneurs privés qui la livrent en camion à des prix au moins dix fois supérieurs à celle dont bénéficient les classes moyennes.

De plus, c'est faire la preuve d'un véritable pessimisme technologique. En effet, mettre à plat des normes techniques communément acceptées (mais généralement élaborées dans de tout autres contextes) et se demander si on ne peut pas significativement abaisser les coûts en faisant preuve de plus de souplesse et d'imagination, c'est en vérité une véritable source d'innovations pour l'ensemble des techniques de fourniture de services essentiels. L'innovation vient toujours de la nécessité de contourner une contrainte, en l'occurrence celle du coût maximum supportable par le client. Il est à notre avis certain que des innovations transférables dans les pays riches eux mêmes viendront d'une recherche approfondie de solutions à bas coût pour les quartiers pauvres du tiers monde.

Dans ce domaine, deux types d'expériences nous semblent particulièrement intéressantes : les réseaux condominiaux, principalement développés en Amérique latine, et les programmes de la Lydec au Maroc

\section{Les réseaux condominiaux.}

Élaboré dans les années 80 dans l'État de Rio Grande do Norte par José Carlos Melo pour les communautés à faible revenu, le modèle condominial d'extension des réseaux est désormais la norme pour des zones urbaines entières du Brésil, quel que soit le revenu des résidents.

Il repose sur une organisation communautaire à l'échelle d'un groupement d'habitation, le condominium. L'infrastructure située au-delà du connecteur secondaire du condominium jusqu'aux stations de traitement et d'épuration est le réseau public. Les investissements et l'exploitation de ce réseau relèvent d'un service public, qui est financé grâce aux redevances payées par les usagers. Dans le condominium, la coordination entre les résidents permet de faire passer le réseau au sein même des lots d'habitation plutôt qu'au milieu de la voirie, ce qui est normalement pratiqué. Cette configuration originale du point de vue technique et organisationnel permet à la fois de réduire les longueurs et les diamètres de réseaux, le nombre de regards et la profondeur d'enfouissement des tuyaux. La population peut également être impliquée dans le creusement des tranchées et la maintenance du réseau. 
En dehors du Brésil, les réseaux condominiaux ont été expérimentés dans la ville de Karachi, où des réseaux d'assainissement condominial ont été mis en place à partir de 1985 dans le plus grand bidonville de la ville, Orangi. Plus récemment, la société Aguas de Illimani a expérimenté l'extension condominiale de ses réseaux d'eau et d'assainissement à El Alto, faubourg de La Paz en Bolivie. Cette expérience a fait l'objet d'une évaluation économique approfondie (Foster, 2001) dont les résultats essentiels sont les suivants.

On observe une réduction des coûts d'extension des réseaux de $40 \%$ dans le cas de l'approvisionnement en eau et de $24 \%$ dans le cas du réseau d'assainissement liquide.

En plus de la réduction des coûts liés à des standards "adaptés » par rapport au modèle conventionnels, la participation des populations à l'extension du réseau permet de faire baisser encore le coût de $12 \%$ dans le cas de l'eau et de $24 \%$ dans le cas de l'assainissement. Ces économies se décomposent ainsi :

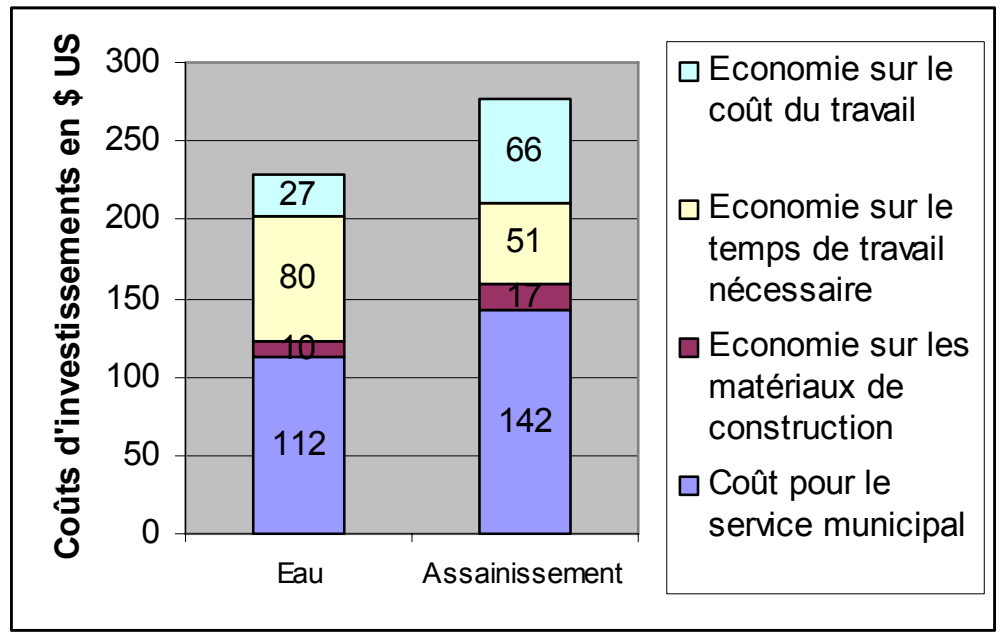

Les économies liés à la participation aux travaux des habitants doivent être confrontées au coût de cette participation, estimée à partir d'une estimation du temps consacrés par les habitants aux activités de formation, de sensibilisation, et de travaux. Dans le cas d'El Alto, le coût d'opportunité du temps était estimé à $2.5 \$$ jour contre un salaire journalier de $6 \$$ jour pour un ouvrier salarié du BTP. Le bilan d'ensemble est alors le suivant :

$\begin{array}{lll}\text { Economies sur le } & \text { Coût d'opportunité du } & \text { Bénéfice net de la } \\ \text { coût du travail } & \text { temps } & \text { participation }\end{array}$

$\begin{array}{llll}\text { Eau } & 27 & 27 & 0\end{array}$

Assainissement 66

30

36

C'est donc surtout pour l'assainissement que la participation des habitants est socialement 
efficace.

Sur la base du cas d'El Alto, on peut retenir que les réseaux condominiaux permettent une économie d'investissement de branchement de l'ordre de 50\% pour le prestataire des services d'eau et d'assainissement, au prix d'un abaissement de la sévérité des normes techniques et d'une certaine participation en travail des habitants.

\section{Le programme INDH à Casablanca}

Sous l'injonction du décret royal concernant l'INDH au Maroc, la Lydec, qui est opérateur des services d'eau, d'assainissement et d'électricité à Casablanca, a du concevoir et chiffrer un programme d'extension à tous des services dont elle est responsable. Pour ce qui est de l'électricité, elle avait déjà adopté, depuis plusieurs années, une méthode « condominiale » dans les quartiers les plus pauvres sous sa responsabilité. Lydec pose un compteur de tête de rue et une organisation privée, sous la responsabilité de l'un des habitants de la rue, se charge de raccorder les familles en aval du compteur principal (chaque famille ayant le sien), selon des normes simplifiées mais approuvées et contrôlées par Lydec, et d'assurer le recouvrement des factures. Le taux de recouvrement des factures est de 97,5\%, supérieur à celui des branchements individuels directs dans le reste de la ville.

Pour l'eau, Lydec prévoit des branchements individuels en polyéthylène soudé, sans enfouissement sauf au passage des rues, et une collecte des eaux grises en PVC de diamètre réduit et en tranchées peu profondes. Pour les toilettes, les fosses simples, vidangées périodiquement par le soin des habitants, resteraient en service dans un premier temps. Des solutions d'assainissement local sont à l'étude pour les zones éloignées du réseau existant. Le coût total du programme pour les 150000 ménages à équiper de Casablanca (soit 1 million d'habitants), serait de 200 millions $€$ financés ainsi :

Financement du programme INDH à Casablanca

\begin{tabular}{|r|r|}
\hline \multicolumn{1}{|l|}{ Gestion déléguée, dont : } & $51 \%$, dont : \\
\hline Collectivités locales & $5 \%$ \\
\hline Opérateur (Lydec) & $7 \%$ \\
\hline Be prénéficiaires & $39 \%$ \\
\hline $\begin{array}{l}\text { Fonds de solidarité du Ministère de l'habitat (reversement d'une partie de } \\
\text { la taxe prélevée sur l'ensemble des factures d'eau pour l'extension des }\end{array}$ & $9 \%$ \\
\hline
\end{tabular}




\begin{tabular}{|l|l|}
\hline réseaux) & \\
\hline $\begin{array}{l}\text { ONEP (Office National de l'Eau Potable, fournisseur d'eau brute aux } \\
\text { distributeurs) }\end{array}$ & $9 \%$ \\
\hline Subventions, en particulier internationales, à mobiliser.... & $31 \%$ \\
\hline
\end{tabular}

Source : Martine Villars, Lydec, 2006. (Villars, 2006)

Le cas est intéressant car il montre, selon les analyses très minutieuses de Lydec sur les capacités à payer des habitants des bidonvilles, que ceux-ci, malgré le coût réduit du programme, ne pourraient cependant qu'en financer 39\% par leurs factures, alors même qu'ils parviennent sans difficulté à supporter le coût total des branchements et de la consommation électrique. Les subventions directes et croisées internes au Maroc seraient de 30\% et le reste, soit $31 \%$, est attendu de l'aide internationale.

Sur cette base de cette évaluation on voit qu'avec des techniques adaptées, les services essentiels de l'eau et de l'électricité pourraient être assurés à la totalité du million des habitants les plus pauvres d'un ville comme Casablanca pour un coût total de $200 €$ par habitant et que cela exigerait, pour être financé sans attendre, un transfert international unique de $60 €$ par habitant.

Voilà qui donne une base de calcul sans doute beaucoup plus réaliste que les chiffres qui circulent actuellement sur les transferts nécessaires pour atteindre les «Objectifs de Développement du Millénaire »(ODM). Sachant que dans le cas de l'INDH à Casablanca, il s'agit de servir la totalité de la population et non de réduire simplement de moitié le nombre des non desservis d'ici 2015, comme dans les ODM. Impressionnants sont en effet les chiffres qu'on brandit pour alerter sur le fait qu'on ne parviendra pas, au train ou vont les choses, à atteindre les objectifs pourtant modestes de développement du millénaire. Une revue récente des différentes estimations proposées depuis l'annonce des ODM en 2000 cite des montants annuels d'investissements entre 9 et 30 milliards de US \$ (Toubkiss, 2006). L'étude mentionne également que ces chiffres sont des minimums puisqu'ils omettent les dépenses nécessaires à la réhabilitation des infrastructures existantes, au développement de nouvelles ressources, ainsi qu'au traitement des eaux usées.

La conclusion qui s'impose alors est que les investissements actuellement consacrées au secteur de l'eau et de l'assainissement doivent être augmentés de manière radicale. Ce message, adressé par l'ONU et la Banque Mondiale aux participants du Forum Mondial de l'Eau de Mexico en 2006 reprends celui du Panel International sur le Financement de l'Accès à l'eau (Camdessus, 2003) qui, lors du précédent Forum Mondial de l'Eau à Kyoto, en 2003, appelait à un 
doublement des investissements dans le secteur de l'eau et de l'assainissement, aujourd'hui évalués entre 14 et 16 Milliards de US \$ par an.

Donc, très grossièrement, il faudrait investir 15 milliards de US \$ pendant 15 ans pour servir environ 500 millions d'individus, soit 450 US \$ par habitant, un chiffre très supérieur à celui annoncé par la Lydec pour Casablanca (200€ par habitant). Remarquons que cette réduction de $40 \%$ des chiffres de la Lydec par rapport à ceux du rapport Camdessus est du même ordre de grandeur que la réduction de coût mesurée dans le cas du réseau condominial d'El Alto à La Paz. On peut donc soupçonner que les chiffres globaux qui circulent actuellement sont surestimés, parce qu'on n'a pas pris en compte les effets de «l'adaptation technologique ».

\section{5) Les riches font sécession : est ce si grave?}

\section{Lutter contre l'automobile en ville}

On a vu que face à la mauvaise qualité des services municipaux, des quartiers résidentiels entiers et des établissements industriels s'équipaient eux même, en général en puisant dans la nappe pour ce qui est de l'eau et en produisant eux même leur électricité. L'équipement croissant en automobile des riches et leur pression constante sur les municipalités pour équiper la ville pour l'automobile (les «flyovers», viaducs qui enjambent les croisements de rues principales, et les ceintures concentriques de périphériques se multiplient dans les villes du sud sans pour autant parvenir à réduire une congestion croissante) peuvent aussi être considérés comme des stratégies de sécession, puisque les pauvres n'ont pas accès à l'automobile, mais supportent quand même les embouteillages dans leurs bus bondés et déglingués, taxis collectifs bringuebalants et autres « rickshaws », sans compter que la voiture a tendance à chasser le vélo et même la marche à pied de la ville.

Pour ce qui est du transport, cette évolution est dramatique du point de vue de l'effet de serre. Mais la solution existe: équiper la ville en moyens de transports rapides de masse (TRM) qui soient assez confortables pour que les propriétaires de voitures ne répugnent pas à les utiliser et néanmoins financièrement accessibles aux pauvres. Une fois que cette offre de transport existe, on peut s'attaquer fermement à la réduction de l'usage de l'automobile, en la taxant ou en limitant son espace de circulation. Le Bus Rapid Transit (BRT) est certainement la meilleure solution quand le métro ne se justifie pas encore. Le BRT est un système de bus articulés de grande capacité (160 personnes à Bogota) circulant en site propre. L'investissement initial est de 10 à 20 fois inférieur à celui d'un métro lourd, alors que la capacité peut atteindre de l'ordre de 30000 personnes par heure, soit le tiers de celle des métros les plus saturés (Tokyo et Hong 
Kong). Cependant, les politiques d'offre de TRM, tels que métros et BRT, doivent impérativement s'accompagner de politiques d'urbanisme adaptées. Car en rendant la ville plus accessible, elles favorisent aussi l'étalement urbain le long et autour de leurs axes. Les POS (Plans d'Occupation des Sols) et COS (Coefficient d'Occupation des Sols) doivent donc être adaptés pour faciliter la concentration des investissements privés dans les zones de forte accessibilité engendrées par la construction d'un TRM et pour diminuer le besoin de mobilité grâce a une densité et une mixité des fonctions urbaines plus fortes. Associer ainsi politique de transport et politique d'usage des sols est naturellement beaucoup plus facile à dire qu'à faire, ce dont témoigne la situation générale de non maîtrise de l'évolution des formes urbaines qui prévaut aujourd'hui, même en présence de politiques de transport de masse vigoureuses (Giraud, Lefevre 2006).

\section{Encadrer les innovations dans les systèmes décentralisés}

En première analyse, les stratégies de sécession des riches dans les domaines de l'eau et de l'électricité sont également néfastes, cette fois parce qu'elles permettent d'échapper aux subventions croisées via la tarification des services municipaux. Mais en vérité, il y a d'autres moyens de taxer les riches, comme la taxe foncière, et d'autre part, il est fort possible que des stratégies, qui au départ sont vécues comme des stratégies de survie, permettent à la fin d'inventer de nouveaux systèmes décentralisés moins coûteux, si l'on prend en compte les coût totaux (coût environnementaux compris), que les systèmes traditionnel en réseau. Ces systèmes décentralisés «modernes », qui d'ailleurs retrouvent en général des principes très anciens tels que la collecte des eaux de pluie, l'assainissement biologique local, la recharge des nappes, etc., pourraient, grâce au progrès techniques et aux effets de série, s'avérer globalement moins coûteux que l'extension des réseaux centralisés.

Dans ces conditions, loin de chercher à entraver ces initiatives privées, la puissance publique devrait se contenter de les réglementer sur le plan environnemental, en particulier de réglementer l'usage de l'eau souterraine, et d'encourager le développement d'une offre de qualité par les industriels locaux. Si, comme c'est probable, cela conduit à la généralisation de systèmes décentralisés complémentaires des réseaux classiques, environnementalement plus efficaces et moins coûteux, les pauvres finiront aussi par en bénéficier.

6) Fiscalité locale et financement des infrastructures de services essentiels 
On l'a vu, les plus pauvres ne peuvent pas toujours supporter le coût complet des services urbains essentiels, même après avoir fait sauter le tabou de l'égalitarisme technologique. Le point dur semble être l'assainissement, ce qui explique d'ailleurs que c'est de loin le domaine où la situation est la plus mauvaise dans les villes du sud. Des transferts internes aux habitants de la ville sont donc nécessaires.

En raison des stratégies de sécession, les subventions croisées par les tarifs ne peuvent excéder un niveau limité. Si elles sont trop fortes, elles incitent les gros consommateurs, qui sont bien sûrs les plus rentables pour le distributeur, à échapper au service public en réseau. Les limites aux subventions croisées augmenteront si les systèmes alternatifs voient leurs coûts baisser, ce qui est en cours.

On peut cependant noter que la vielle méthode des «Stadtwerke » allemands (subventions croisées entre services) ne devrait pas être négligée dans les villes du sud. Le consentement à payer des riches et même des pauvres pour une électricité distribuée par réseau est en général plus élevé que celui pour l'assainissement par exemple, et les stratégies de sécession peuvent être plus coûteuses ou impossibles dans l'électricité. Si bien qu'on peut envisager de subventionner l'assainissement en taxant l'électricité. C'est ce qui se passe, semble-t-il, dans certaines municipalités sud africaines et certaines grandes villes marocaines, par exemple.

Mais reste fondamentalement le recours à la fiscalité locale générale. En la matière, tous les fiscalistes affirment qu'un bon système fiscal doit être «simple et stable». Dans les villes du sud, le meilleur candidat est sans conteste la taxe annuelle sur la valeur vénale des propriétés foncières et bâties. Un taxe foncière exige un cadastre ou du moins un support d'information foncière s'en rapprochant. Et c'est là que la situation est très différente au nord et au sud. Elaborer un cadastre pose immédiatement la question ardue de la définition et de l'attribution de droits de propriété sur le foncier et le bati. Ces droits sont en effet, dans la plupart des villes du sud, très complexes. On trouve une juxtaposition de systèmes traditionnels et importés, et des droits d'extension très variée par rapport à la simplicité théorique du droit romain et même du droit anglosaxon. Naturellement dans ces villes, on l'a dit, beaucoup occupent et ont bati sans aucun droit, ni coutumier ni moderne, en particulier sur des terrains publics.

Il est facile d'affirmer, comme le font un grand nombre d'experts des agences de developpement, que la priorité dans les villes du sud est de définir des droits de propriété, puis de les attribuer à des propriétaires publics ou privés, de manière à ce que les marchés fonciers et immobiliers fonctionnent et que les autorités publiques puissent les influencer par des réglementations et des taxes. C'est certainement vrai en théorie. Mais pour cela, il faut préciser 
les droits fonciers et les enregistrer dans un cadastre. Or dans la pratique, selon Vincent Renard (Renard 2002), les cadastres et les systèmes juridiques d'application des droits de propriétés urbains longuement mis en place dans la villes riches, et qui sont aujourd'hui proposés aux villes du sud par les consultants en developpement, ont une sophistication qui induirait des temps de mise en place tels que les cadastres ne seraient pas terminés avant la fin de l'actuelle phase d'expansion urbaine rapide. L'élaboration d'un cadastre sophistiqué peut ainsi être un frein au développement de la propriété. En matière de légalisation et d'enregistrement de la propriété foncière, il faut faire beaucoup plus simple, au prix d'approximations qui se régleront dans le temps.

Ces critiques ne sont pas restées totalement sans écho. On peut noter par exemple que la technique simple de l' "adressage » a été développée dans un certain nombre de pays, en particulier dans des programmes de la Banque Mondiale. L'adressage consiste à constituer un fichier de la population urbaine au départ très simple, puisque l'«information racine» est simplement l'adresse. On peut ensuite, selon les besoins et les capacités de collecte de l'information, lui ajouter progressivement un grand nombre de données (type de voirie ou de bâtiments, superficie, numéro de compteur d'eau, ...) utiles à la municipalité dans la formulation et la mise en œuvre de sa politique urbaine, y compris de sa politique fiscale.

Mais une voie plus originale est explorée de manière croissante, en Amérique latine en particulier. Il s'agit d'une forme particulière de taxation des plus values liées aux infrastructures de services, permettant d'en financer l'extension. Une taxation systématique des plus values immobilières et foncières au moment des transactions est, de l'avis des spécialistes, impraticable dans les grandes villes du sud où les titres de propriété sont complexes ou inexistant, et où les marchés immobiliers et fonciers, en particulier dans l'informel, sont très opaques. En revanche, taxer les propriétaires dès le moment où une infrastructure publique particulière (voirie goudronnée, drainage, arrivée du réseau d'eau et d'assainissement, arrivée d'un TRM donnant accès plus rapidement et à moindre coût au centre ville) a augmenté sans conteste la valeur de marché de leur propriété est une voie prometteuse, car plus facile d'application et moins sujette à contournement et corruption. C'est le cas en particulier pour les infrastructures de transports, et c'est ainsi que certaines municipalités sud américaines envisagent de les financer.

L'essence de ce type de mesure est de maximiser, dans le temps, le taux de collecte du consentement à payer des consommateurs. La fourniture d'un service essentiel valorisant, toutes choses égales par ailleurs, un droit de propriété foncier ou immobilier (si ce droit, dans un cadre juridique traditionnel ou moderne, est reconnu), les ayant droits sont en général prêts à en assumer, à ce titre, au moins une partie du coût. Mais il faut qu'ils puissent le faire sur la base 
de leurs revenus actuels, alors qu'ils n'ont pas encore réalisé, et ne réaliseront peut être pas avant longtemps, leur plus value foncière. On voit donc que la mise en place de ces formes spécifiques de taxation des plus values liées aux infrastructures de services doit s'accompagner d'une amélioration de l'accès au crédit, voire au micro crédit, des consommateurs.

\section{Conclusions}

L'accès de tous aux services urbains essentiels semble aujourd'hui un objectif très lointain, car on voit mal comment on pourrait le financer. Dans cet article, nous avons voulu tempérer ce pessimisme, sur la base de l'analyse d'un certain nombre de cas qui permettent à la fois de bien identifier la nature des problèmes et de mieux les quantifier.

Il apparaît tout d'abord que le coût total sera certainement inférieur aux évaluations actuelles, si on ose aller dans la voie de l'innovation technologique et organisationnelle que constituent des systèmes adaptés aux quartiers pauvres. Si l'on veut aller vite, il reste vrai que des transferts, non seulement internes à la ville et au pays, mais internationaux sont nécessaires. Mais leur volume est certainement moindre que ce qu'on annonce généralement.

Pour augmenter rapidement le nombre de pauvres urbains ayant accès à des services essentiels de qualité et cependant adaptés à leurs capacités financières, les problèmes sont cependant loin d'être uniquement financiers. Ils sont tout autant politiques, organisationnels, et techniques. Pour les surmonter, il faut oser mettre en œuvre à grande échelle des innovations qui ont déjà été expérimentées ici ou là et qui y ont prouvé leur intérêt.

Ces innovations portent sur les domaines suivants ; 1) la régularisation des «sans citoyenneté urbaine »,2) la réforme des services publics, qui peut être grandement favorisée par des formes de partenariat public privé adaptées et surtout progressives (pas de privation brutale d'un service public devenu notoirement inefficace, mais son «entreprisation » préalable par des contrats de gestion), 3) la levée du tabou de la solution technique unique pour tous et la mise en œuvre de solutions techniques et organisationnelles qui abaissent les coûts totaux d'accès aux réseaux, 4) la réforme de la fiscalité locale, afin qu'elle capte le maximum du «consentement à payer » pour une amélioration des services essentiels. Pour inventer des solutions aujourd'hui, il suffit dans la plupart des cas de s'interroger avec pragmatisme sur l'adaptation au contexte local de ce qui a déjà été démontré ailleurs.

Reste que les pays les moins avancés (PMA) d'une part, les pays émergents (à croissance très 
rapide) et à revenus intermédiaires d'autre part sont inégalement armés pour mettre en œuvre ces solutions. Les pays émergents et intermédiaires 1) peuvent mobiliser des consentements à payer plus élevés des consommateurs, 2) peuvent organiser des transferts internes plus importants, et avoir accès plus facilement à des ressources financières longues, 3) possèdent déjà des moyens humains et des institutions qui rendent plus facile ou simplement possible la réforme des services publics inefficaces et les innovations organisationnelles dans leur gestion.

Il est donc clair que dans les quartiers les plus pauvres des villes des PMA, pendant encore plusieurs décennies, seules seront accessibles les solutions minimales, mises en œuvre par le secteur informel ou par des ONG avec l'aide des habitants concernés et en partie financées par l'aide internationale. Pour l'eau par exemple : la borne fontaine et des toilettes améliorées par rapport au simple trou dans la terre, gérées par des associations de quartier ou le secteur informel local.

On peut en conséquence se demander s'il ne faudrait pas réserver aux PMA l'aide publique au développement dans ces secteurs. C'est certainement juste sur le fond, mais l'aide publique devrait aussi sans hésiter soutenir les solutions innovantes qui émergent en général dans des pays intermédiaires, par exemple en Amérique latine ou au Maroc, afin que ces expériences, potentiellement utiles à tous, puissent être validées plus rapidement et servir ensuite d'exemples au reste du monde. 


\section{Références}

AFD, 2005, "Financer les investissements des villes des pays en developpement ", Notes et document $\mathrm{N}^{\circ} 24$, Décembre 2005

Camdessus, 2003, "Financer l'eau pour tous, Rapport du panel mondial sur le financement des infrastructures de l'eau », présenté au $3^{\text {ème }}$ Forum Mondial de l'eau, Kyoto, 2003

Cour, J.-M. 2005, : Investissements de peuplement et d'urbanisation, besoins de financement et implications pour la coopération internationale ». Téléchargeable sur le site de 1'IDDRI : http://www.iddri.org/iddri/telecharge/services/05_6_cour.pdf

Conan, H., 2004 Small Piped Water Networks : Helping Local Entrepreneurs to Invest Water For All Series, n. 13, Asian Development Bank

Dutta et. al. (2005) Public support for water supply improvements: Empirical evidence from Unplanned settlements of Delhi, India. The Journal of Environment Development. 2005; 14: 439-462

Foster, V., 2001 Economic and financial evaluation of El Alto Project: Condominial water and sewerage projects and related innovations. Washington, the World Bank Group.

Giraud, Lefèvre 2006 "Les défis énergétiques de la croissance urbaine au sud Le couple "Transport - Urbanisme» au cœur des dynamiques urbaines", in AFD/IDDRI, " Regards sur la Terre », Presses de la Fondation Nationale des Sciences Politiques, Paris, octobre 2006

Levasseur J., Maria A., (2004) "Strategies for water supply in a planned urban extension: A case study of the Dwarka sub-city in Delhi", Research Report, Cerna, September 2004. http://www.cerna.ensmp.fr/Documents/AM-LJ-Dwarka.pdf

Llorente M. et Zérah M.-H.,2003,"Urban Water Sector: Formal versus Informal Suppliers in India", Urban India, Vol. XXII, no 1, New Delhi, National Institute of Urban Affairs, January-June, 2003.

Maria A. (2004) "The role of groundwater in Delhi's water supply" Présentation au cours d'été en Hydrogéologie urbaine, organisé par l'OTAN à Baku, Aout 2004, http://www.cerna.ensmp.fr/Documents/AM-GroundwaterDelhi.pdf

Maria A. (2005) "La crise de l'approvisionnement en eau à Delhi. Réponse des acteurs et scénarios d'évolution" Présentation au séminaire Accés aux Services Essentiels de l'IDDRI, à Paris, Avril 2005,

http://www.iddri.org/iddri/html/services/sem_services.htm

Maria Augustin, Ruet Joël, Zérah Marie-Hélène, Giraud Pierre-Noël, "Infrastructures 
urbaines de l'eau en Inde : quels enjeux?', Les Notes de l'IDDRI, "Eau : encadrer les partenariats public-privé", IDDRI, 2003,

\section{http://www.iddri.org/iddri/telecharge/notes/02-eau.pdf}

Renard, V., 2002, «Les dynamiques économiques des villes du $21^{\text {ème }}$ siècle : dérives des marchés immobiliers et fragmentation urbaine ». Communication à l'Académie des Sciences Morales et Politiques.

Ruet J. (2005) "Privatising Power cuts? Ownership and organisational reform of State Electricity Boards in India”, 339p, Academic Foundation, New Delhi.

Ruet J. (2006). "The Real Option Approach to Privatise a Utility Where Information is Scarce, and management contract as a limit case", forthcoming LSE-DESTIN Working Paper $n^{\circ} 74$, Papers Series, Development Studies Institute, LSE, London.

Ruet J. (2006b). "Cost-Effectiveness of Alternative Investment Strategies for the Power Sector in India: A Retrospective Account of the Period 1997-2002", forthcoming 2006, Utilities Policy

Ruet J., Saravanan V.S., Zérah M.H.,2002 "The Water and Sanitation Scenario in Indian Metropolitan Cities: Resources and Management in Delhi, Calcutta, Chennai, Mumbai", Occasional Paper no 6, Centre de Sciences Humaines, New Delhi, 2002.

Toubkiss, J., 2006, «Objectifs du Millénaire pour le développement. Evaluer le coût d'atteinte de la cible 10 sur l'eau et l'assainissemen ». Conseil Mondial de l'Eau. UN, Population Division, Mars 2000, World urbanization prospects: the 1999 revision

Villars, M., 2006, " Habitat précaire et services essentiels, le cas de Casablanca » Présentation au séminaire « Services essentiels » de IDDRI/Cerna:

http://www.iddri.org/iddri/html/services/sem_services.htm 